\title{
Establishing a memory-dependent photothermoelastic model according to the dual phase lag model on a cylindrical body rotating in an initial magnetic field
}

\author{
Kadry Zakaria ${ }^{1}$, Magdy Sirwah ${ }^{1}$, Ahmed Abouelregal ${ }^{2}$, and Ali Farouk Rashid ${ }^{1}$ \\ ${ }^{1}$ Tanta University \\ ${ }^{2}$ Mansoura University Faculty of Science
}

January 29, 2021

\begin{abstract}
This paper deals with the study of photothermoelastic interactions in an isotropic homogeneous semiconductor solid, using a new model of generalized thermoelectricity with a memory-dependent derivative of heat conduction. The plasma and thermal effects study of semiconductor structures include the simulation of a complex system using simultaneous analysis of carrier density, thermal waves, and elastic wave equations. On this topic, there are few research works that have been achieved. To investigate the problem, Tzou's generalized theory is employed. The governing equations of the system are derived based on the dual-phase lag model (DPL) and the wave equation of coupled plasma. We examined the transient response of a rotating solid cylinder subjected to the applied magnetic field and a time-dependent heat flow under the new proposed model. The analytical expressions for the investigated fields are derived by using the Laplace transform process and the numerical results are graphically previewed. A comparison of the numerical results is provided for various models of thermoelasticity as well as the impact of memory- dependent derivatives. The effects of rotation, time-delay and the kernel function are also investigated
\end{abstract}

\section{Hosted file}

Final Manuscript.pdf available at https://authorea.com/users/392505/articles/506376establishing-a-memory-dependent-photothermoelastic-model-according-to-the-dual-phaselag-model-on-a-cylindrical-body-rotating-in-an-initial-magnetic-field 
figures/Figure-1/Figure-1-eps-converted-to.pdf 
figures/Figure-2/Figure-2-eps-converted-to.pdf 
figures/Figure-3/Figure-3-eps-converted-to.pdf 
figures/Figure-4/Figure-4-eps-converted-to.pdf 
figures/Figure-5/Figure-5-eps-converted-to.pdf 
figures/Figure-6/Figure-6-eps-converted-to.pdf 
figures/Figure-7/Figure-7-eps-converted-to.pdf 
figures/Figure-8/Figure-8-eps-converted-to.pdf 
figures/Figure-9/Figure-9-eps-converted-to.pdf 
figures/Figure-10/Figure-10-eps-converted-to.pdf 
figures/Figure-11/Figure-11-eps-converted-to.pdf 
figures/Figure-12/Figure-12-eps-converted-to.pdf 
figures/Figure-13/Figure-13-eps-converted-to.pdf 
figures/Figure-14/Figure-14-eps-converted-to.pdf 
figures/Figure-15/Figure-15-eps-converted-to.pdf 
figures/Figure-16/Figure-16-eps-converted-to.pdf 